DOI https://doi.org/10.30525/978-9934-26-008-7.2-5

\title{
INFLUENCE OF COBALT SUBSTITUTION \\ ON THE STRUCTURE AND PHYSICAL PROPERTIES OF SUBSTITUTED LITHIUM IRON SPINEL
}

\section{Mazurenko Yu. S., Kaykan L. S.}

\section{INTRODUCTION}

In this work, nanoparticles of lithium spinel ferrites $\left(\mathrm{Li}_{0.5-\mathrm{x} / 2} \mathrm{Co}_{\mathrm{x}} \mathrm{Fe}_{2.5-}\right.$ $\left.{ }_{\mathrm{x}} \mathrm{O}_{4}, \mathrm{x}=0.0 ; 0.2 ; 0.4 ; 0.6 ; 0.8 ; 1.0\right)$ were successfully synthesized by a modified method of sol-gel auto-combustion. Lithium nitrate, cobalt nitrate and iron nitrate were selected as the source of monovalent and divalent $\left(\mathrm{Li}^{+} \mathrm{i} \mathrm{Co}^{2+}\right)$ and trivalent $\left(\mathrm{Fe}^{3+}\right)$ cations, respectively, and as a reducing agent (fuel) selected citric acid. It was studied the influence of cobalt ions on the morphology, structural, electrical and magnetic properties of $\mathrm{Li}_{0.5-\mathrm{x} / 2} \mathrm{Co}_{\mathrm{x}} \mathrm{Fe}_{2.5-\mathrm{x}} \mathrm{O}_{4}$ spinel. To study the phase purity, microstructure, particle size, elemental composition, determine the functional group, calculate the size of the crystallites, magnetic properties and the influence of synthesis conditions, in particular the $\mathrm{pH}$ of the reaction medium, various methods were used: X-ray diffraction (XRD), high-resolution scanning electron microscopy (HR-SEM), energy-dispersing X-ray spectroscopy (EDX) and vibration magnetometer (VSM). The observed results showed that the final product is a cubic spinel with a spherical morphology of nanoparticles. Moreover, with increasing cobalt concentration in lithium ferrites synthesized by the sol-gel method of auto combustion, the saturation magnetization increases to $\mathrm{x} \leq 0.8$, and then decreases. This is because $\mathrm{Co}^{2+}$ ions with high magnetic moment replace non-magnetic $\mathrm{Li}^{+}$ions in synthesized Li-Co ferrite samples.

\section{Optimization of structure of nanosized lithium ferrites obtained} by sol-gel auto-combustion method by substitution of cobalt ions

Polycrystalline nanosized ferrites have good chemical stability and high magnetic characteristics, which depend on a number of factors, such as method of preparation, type and amount of replacement element, heat 
treatment, etc. ${ }^{1}$. Lithium and substituted lithium ferrites with a spinel structure have proven to be a good substitution of garnets in microwave devices due to their low cost, high resistance, low eddy current losses ${ }^{2}$ and at the same time high magnetic characteristics, such as saturation magnetization, residual magnetization, high Curie temperature ${ }^{3}$.

The traditional method of obtaining spinel ferrites - ceramic synthesis has a number of disadvantages, one of the main is the high temperature (about $1000-1200^{\circ} \mathrm{C}$ ) and long sintering time, which has a particularly negative effect on the quality of lithium ferrite. There are a sufficient number of chemical methods that allow synthesis at relatively low temperatures and thus prevent the loss of lithium in the final product. These include coprecipitation methods ${ }^{4}$, hydrothermal method ${ }^{5}$ and a number of others. Of great interest in this regard is the method of sol-gel auto-combustion, which at low energy consumption (heat, required for the synthesis reaction, is released during the exothermic interaction of nitric acid residue and chelating agent, which can be citric acid) provides high chemical purity and homogeneity of the final product. In the literature there are works in which studies were carried out on the

1 De Fazio E., Bercoff P.G., \& Jacobo S.E. Electromagnetic properties of manganese-zinc ferrite with lithium substitution. Journal of Magnetism and Magnetic Materials. 2011. No. 323(22), pp. 2813-2817. URL: https://doi.org/10.1016/ j.jmmm.2011.06.022.

${ }^{2}$ Liu J., Mei Y., Liu W., Li X., Hou F., \& Lu G.-Q. Effects of sintering temperature on properties of toroid cores using $\mathrm{NiZnCu}$ ferrites for power applications at $>1 \mathrm{MHz}$. Journal of Magnetism and Magnetic Materials. 2018. No. 454, pp. 6-12. URL: https://doi.org/10.1016/j.jmmm.2018.01.031.

${ }^{3}$ Singh Yadav R., Kuřitka I., Havlica J., Hnatko M., Alexander C., Masilko J., Kalina L., Hajdúchová M., Rusnak J., \& Enev V. Structural, magnetic, elastic, dielectric and electrical properties of hot-press sintered Co1-xZnxFe2O4 (x = 0.0, 0.5) spinel ferrite nanoparticles. Journal of Magnetism and Magnetic Materials. 2018. No. 447, pp. 48-57. URL: https://doi.org/10.1016/j.jmmm.2017.09.033.

${ }^{4}$ Han D., Zhang J., Liu P., Li G., An L., \& Wang S. Preparation of high-quality transparent Al-rich spinel ceramics by reactive sintering. Ceramics International. 2018. No. 44(3), pp. 3189-3194. URL: https://doi.org/10.1016/j.ceramint.2017.11.089.

${ }^{5}$ Rahaman Md.D., Nusrat T., Maleque R., \& Hossain A.K.M.A. Investigation of structural, morphological and electromagnetic properties of $\mathrm{Mg} 0.25 \mathrm{Mn} 0.25 \mathrm{Zn} 0.5-$ xSrxFe2O4 ferrites. Journal of Magnetism and Magnetic Materials. 2018. No. 451, pp. 391-406. URL: https://doi.org/10.1016/ j.jmmm.2017.11.066. 
substitution of ferrites by di- and trivalent elements ${ }^{6,7,8}$, but to date there are no systematic studies of the effect of substitution on morphology, structure and through them on electrical and magnetic properties.

In the given work, the complex analysis of influence of substitution of cobalt ions on structural, electric and magnetic properties of the nanosized ferrites received by a method of sol-gel of autoburning is carried out.

Nanoparticles of $\mathrm{Li}_{0.5-\mathrm{x} / 2} \mathrm{Co}_{\mathrm{x}} \mathrm{Fe}_{2.5-\mathrm{x}} \mathrm{O}_{4}$ composition, where $\mathrm{x}=0.0 ; 0.2 ; 0.4$; $0.6 ; 0.8 ; 1.0$, were obtained by the method of sol-gel auto-combustion using stoichiometric quantities of $\mathrm{LiNO}_{3}, \mathrm{Fe}\left(\mathrm{NO}_{3}\right)_{3} \cdot 9 \mathrm{H}_{2} \mathrm{O}, \mathrm{Co}\left(\mathrm{NO}_{3}\right)_{2} \cdot 6 \mathrm{H}_{2} \mathrm{O}$ and citric acid as a chelating agent. The nitrates of the starting compounds were dissolved in distilled water and mixed on a magnetic stirrer. To the resulting mixture was added citric acid in a ratio of $1: 1.75$ to the total number of metal ions. To ensure the desired $\mathrm{pH}$ value of the reaction solution (7) in the finished mixture of precursors with constant stirring was added an aqueous solution of ammonia. The resulting solution was placed in an oven until complete removal of water and the formation of a xerogel. The xerogel was heated to a temperature of $240^{\circ} \mathrm{C}$. At this temperature, it ignited and after combustion, the final product was obtained. There was no additional annealing of the formed compound.

Sintered powder to establish phase purity and crystallinity was investigated on a diffractometer Rigaku Ultima-IV in $\mathrm{Cu} \mathrm{K}_{\alpha}$ radiation at temperature $293 \mathrm{~K}$. The average crystallite size was determined by Debye Scherrer's formula:

$$
t=\frac{0.94 \lambda}{\beta \cos \theta}
$$

${ }^{6}$ Sun L., Guo J., Zhang R., Cao E., Zhang Y., Hao W., \& Ju L. (2018). Influence of $\mathrm{Cu} 2+$ doping on the structure, dielectric and magnetic properties of $\mathrm{NiFe} 2 \mathrm{O} 4$ prepared by the sol-gel method. Journal of Magnetism and Magnetic Materials. 2018. No. 449, pp. 545-551. URL: https://doi.org/10.1016/j.jmmm.2017.10.104.

${ }^{7}$ Elshahawy A.M., Mahmoud M.H., Makhlouf S.A., \& Hamdeh H.H. Role of $\mathrm{Cu} 2+$ substitution on the structural and magnetic properties of Ni-ferrite nanoparticles synthesized by the microwave-combustion method. Ceramics International. 2015. No. 41(9), pp. 11264-11271. URL: https://doi.org/10.1016/ j.ceramint.2015.05.079.

${ }^{8}$ Ahmed M.A., Mansour S.F., \& Afifi M. Structural and electrical properties of nanometric $\mathrm{Ni}-\mathrm{Cu}$ ferrites synthesized by citrate precursor method. Journal of Magnetism and Magnetic Materials. 2012. No. 324(1), pp. 4-10. URL: https://doi.org/10.1016/j.jmmm.2011.07.010. 
where $\lambda$ - wavelength of $\mathrm{Cu} \mathrm{K}_{\alpha}$ radiation ( $\lambda=1,5406 \AA$ ), $\beta$ - reflex width at half height (FWHM) in radians, as calculated by the Gaussian approximation. The contribution due to instrumental broadening was also taken into account, when calculating the accuracy of the size of the crystallites $^{9}$. In this work, the instrumental widening was installed using a standard silicon sample and by the method of Williamson Hall.

The X-ray density of the synthesized samples was calculated by the ratio (2)

$$
D_{x}=\frac{Z M}{N a^{3}}
$$

where $\mathrm{Z}$ - the number of molecules per formula unit (for ferrites it is 8), M - molecular weight, $\alpha$ - lattice parameter and N - Avogadro's number. Transport properties were calculated from formulas $d_{A-A}=0.25 a \sqrt{2}, d_{B-B}=0.25 a \sqrt{3}$, where $a$ lattice constant.

Cationic distribution as well as other parameters such as lattice constant $a$, oxygen parameter $u$ and half-width of peaks $\beta$ obtained from the analysis of experimental X-ray spectra by the full-profile Rietveld refinement method.

Absorption spectra from ${ }^{57} \mathrm{Fe}$ were obtained on a Mössbauer spectrometer MS1104EM. As a source of $\gamma$-quanta applied 57 Co in the matrix of chromium with activity $100 \mu \mathrm{Ku}$. The decoding of the obtained spectra was performed in the Univem package with calibration relative to $\alpha-\mathrm{Fe}$.

The surface morphology of the samples was examined using a scanning electron microscope FE-SEM QUANTAFEG 250. Measurements were performed at accelerating voltage $15 \mathrm{kV}$.

The study of the conductive and dielectric properties of the samples was carried out using an impedance spectrometer Autolab PGSTAT 12/FRA-2 in the frequency range $0.01 \mathrm{~Hz}-100 \mathrm{kHz}$.

9 Sijo A.K. Influence of fuel-nitrate ratio on the structural and magnetic properties of $\mathrm{Fe}$ and $\mathrm{Cr}$ based spinels prepared by solution self-combustion method. Journal of Magnetism and Magnetic Materials. 2017. No. 441, pp. 672-677. URL: https://doi.org/10.1016/j.jmmm.2017.06.060; M.A. Dar, Dinesh Varshney. Effect of d-block element $\mathrm{Co} 2+$ substitution on structural, Mössbauer and dielectric properties of spinel copper ferrites. Journal of Magnetism and Magnetic Materials. 2017. V. 436. Pp. 101-112. 
In fig. $1(\mathrm{a}, \mathrm{b})$ presents the experimental X-ray diffraction patterns of the synthesized systems. As can be seen from the figure, all the obtained compounds are single-phase spinels, and the system of composition $\mathrm{Li}_{0.5} \mathrm{Fe}_{2.5} \mathrm{O}_{4}, \mathrm{x}=0.0$, (Fig. 1(a)) belongs to the spatial group $\mathrm{P} 4_{3} 32$ [JCPDS No 76-1591], the so-called superordered spinel structure, as evidenced by the presence of superstructural peaks (110), (210) and (211). This superstructure occurs due to the ordered arrangement of lithium and iron ions in a ratio of 1:3 in the crystallographic direction.
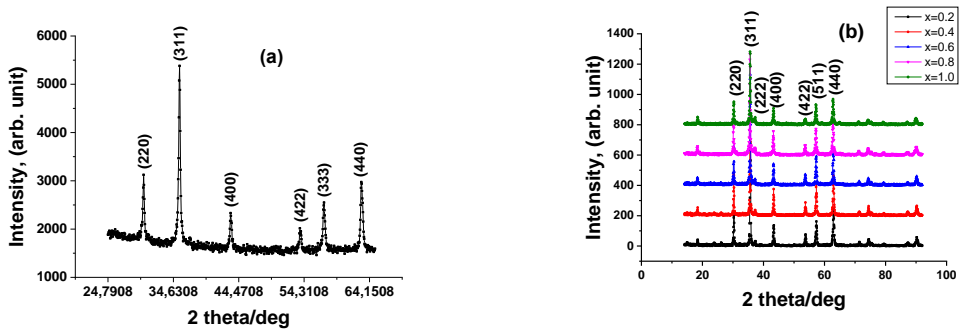

Fig. 1. X-ray diffraction patterns of systems $\mathrm{Li}_{0.5-\mathrm{x} / 2} \mathrm{Co}_{\mathrm{x}} \mathrm{Fe}_{2.5-\mathrm{x}} \mathrm{O}_{4}$ with composition (a) $\mathrm{x}=0.0$; (b) $(x=0.2 ; 0.4 ; 0.6 ; 0.8 ; 1.0)$

Systems $\mathrm{Li}_{0.5-\mathrm{x} / 2} \mathrm{Co}_{\mathrm{x}} \mathrm{Fe}_{2.5-\mathrm{x}} \mathrm{O}_{4}$ composition ( $\mathrm{x}=0.2 ; 0.4$ ) (Fig. 1(b)) also found belonging to an ordered spatial group $\mathrm{P}_{3} 32$, however, starting with $\mathrm{x}=0.6$ the spectra showed the presence of a small amount of disordered phase Fd3m (table 1). Obviously, the presence of cobalt ions in the B-lattice disrupts the order in the arrangement of iron and lithium ions.

Table 1

Phase composition of the system, $\mathrm{Li}_{0.5-\mathrm{x} / 2} \mathrm{Co}_{\mathrm{x}} \mathrm{Fe}_{2.5-\mathrm{x}} \mathrm{O}_{4}$

\begin{tabular}{|c|c|c|c|c|c|}
\hline Cobalt ion content & 0.2 & 0.4 & 0.6 & 0.8 & 1.0 \\
\hline The content of the ordered phase $\mathbf{P 4} \mathbf{3} 32$ & 100 & 100 & 97.07 & 93.56 & 90.55 \\
\hline The content of the disordered phase $\mathbf{~ F d 3 m}$ & 0 & 0 & 2.93 & 6.44 & 9.45 \\
\hline
\end{tabular}

Dependence of a constant lattice on concentration with accuracy up to $\pm 0,002 \AA$, determined by $\mathrm{x}$-ray diffraction patterns for $(x=0.0 ; 0.2 ; 0.4 ; 0.6 ; 0.8 ; 1.0)$ shown in Fig. 2 .

As can be seen from Fig. 2, the lattice parameter increases with increasing concentration of $\mathrm{Co} 2+$, despite the fact that the ionic radius of cobalt $(0.60)$ is smaller than that of iron $(0.64)$ or lithium $(0.72)$, which, 
apparently, may be due to small crystallites and distorting effect of the surface.

The cationic distribution obtained from the analysis of experimental $\mathrm{X}$-ray diffraction patterns using the full-profile Rietveld refinement method is shown in table 2 .

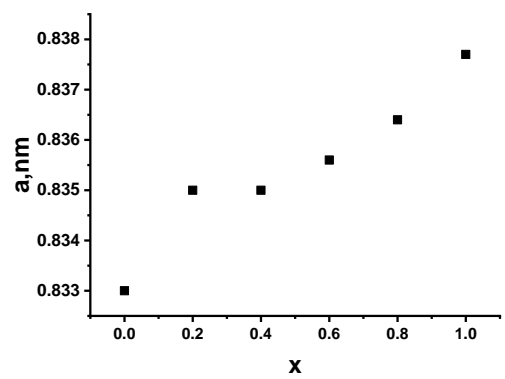

Fig. 2. Dependence of the lattice constant of the system

$\mathrm{Li}_{0.5-\mathrm{x} / 2} \mathrm{Co}_{\mathrm{x}} \mathrm{Fe}_{2.5-\mathrm{x}} \mathrm{O}_{4}$ on the content of cobalt ions

Table 2

Cation distribution on the lattice of the system $\mathrm{Li}_{0.5-\mathrm{x} / 2} \mathrm{Co}_{\mathrm{x}} \mathrm{Fe}_{2.5-\mathrm{x}} \mathrm{O}_{4}$

\begin{tabular}{|c|c|c|c|c|}
\hline$x$ & A-site & B-site & $a . \AA$ & $\Delta a . \AA$ \\
\hline 0.0 & $L i_{0.05} F e_{0.95}$ & $L i_{0.45} F e_{1.55}$ & 8.330 & \pm 0.002 \\
\hline 0.2 & $L i_{0.025} \mathrm{Co}_{0.12} F e_{0.8}$ & $L i_{0.755} \mathrm{Fe}_{1.24} \mathrm{Co}_{0.08}$ & 8.351 & \pm 0.002 \\
\hline 0.4 & $L i_{0,025} C o_{0.24} F e_{0,728}$ & $L i_{0.275} F e_{1.556} C o_{0.16}$ & 8.351 & \pm 0.002 \\
\hline 0.6 & $C o_{0.244} F e_{0.778}$ & $L i_{0.2} F e_{1.401} C o_{0.366}$ & 8.357 & \pm 0.002 \\
\hline 0.8 & $\mathrm{Co}_{0.224} \mathrm{Fe}_{0.717}$ & $L i_{0.1} F e_{1.442} C o_{0.486}$ & 8.364 & \pm 0.002 \\
\hline 1.0 & $\mathrm{Co}_{0.404} \mathrm{Fe}_{0.612}$ & $\mathrm{Fe}_{1.374} \mathrm{Co}_{0.605}$ & 8.377 & \pm 0.002 \\
\hline
\end{tabular}

Table 2 shows that $\mathrm{Li}^{+}$ions occupy mainly the B-sublattice, and $\mathrm{Fe}$ and $\mathrm{Co}$ ions are redistributed by both sublattices. Moreover, with small substitutions $(x \leq 4)$, Cobalt ions mainly occupy the A-sublattice, and with increasing $\mathrm{x}$, the B-sublattice predominates. Fig. 3 shows the distribution of cations by sublattices for different substitution contents. The calculation is given for eight formula units. 


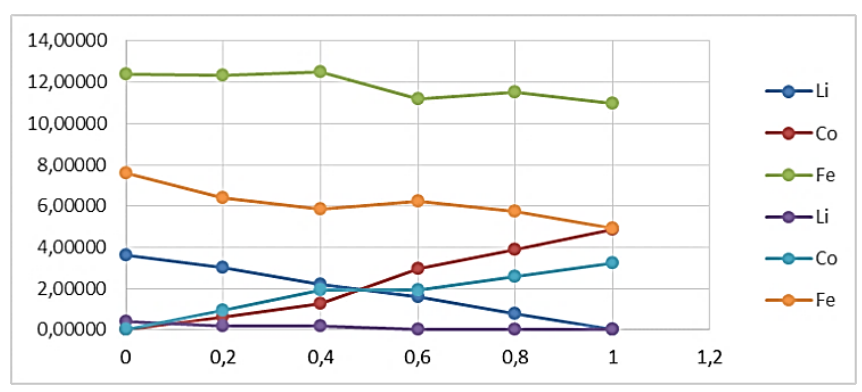

Fig. 3. Distribution of cations on the sublattices of the system $\mathrm{Li}_{0.5-\mathrm{x} / 2} \mathrm{Co}_{\mathrm{x}} \mathrm{Fe}_{2.5-\mathrm{x}} \mathrm{O}_{4}$

Based on the experimentally found values of the lattice constant and the oxygen parameter $(u)$, the values of ionic radii for the $\mathrm{A}$ and $\mathrm{B}$ positions $\mathrm{r}_{\mathrm{A}}$ and $\mathrm{r}_{\mathrm{B}}$ were calculated by the formulas ${ }^{10}$ :

$$
\begin{gathered}
r_{A}=C_{A L i} r L i+C_{A C o} r(C o)+C_{A F e} r\left(F e^{3+}\right) \\
r_{B}=\frac{1}{2}\left[C_{B C o} r\left(C o^{2+}\right)+C_{B F e} r\left(F e^{3+}\right)+C_{B L i} r\left(L i^{+}\right)\right]
\end{gathered}
$$

where $\quad \mathrm{r}\left(\mathrm{Li}^{+}\right), \quad \mathrm{r}\left(\mathrm{Co}^{2+}\right), \quad \mathrm{r}\left(\mathrm{Fe}^{3+}\right) \quad$ ionic radii, and $C_{A C o}, C_{A F e}, C_{A L i}, C_{B C o}, C_{B F e}, C_{B L i}-$ the concentration of the corresponding ions in the $\mathrm{A}$ and $\mathrm{B}$ sublattices. The calculated values of ionic radii for each composition and each sublattice are given in table 3.

Table 3

Experimental and calculated values of the lattice parameter,

\begin{tabular}{|c|c|c|c|c|c|c|}
\hline $\begin{array}{c}\text { Concentration, } \\
x\end{array}$ & $a_{\text {exp }},(\hat{\AA})$ & $a_{t h},(\AA \mathbf{A})$ & $r_{A},(\AA ́)$ & $r_{B},(\AA)$ & $\begin{array}{l}\bar{r}_{r}, \\
(\AA)\end{array}$ & $u,(\AA ̊)$ \\
\hline 0.0 & 8.330 & 8.208 & 0.644 & 0.658 & 0.649 & 0.2600 \\
\hline 0.2 & 8.351 & 8.155 & 0.602 & 0.556 & 0.579 & 0.2477 \\
\hline 0.4 & 8.351 & 8.305 & 0.628 & 0.645 & 0.6373 & 0.2481 \\
\hline 0.6 & 8.356 & 8.316 & 0.644 & 0.621 & 0.633 & 0.2569 \\
\hline 0.8 & 8.365 & 8.327 & 0.653 & 0.643 & 0.648 & 0.2552 \\
\hline 1.0 & 8.377 & 8.322 & 0.634 & 0.621 & 0.628 & 0.2547 \\
\hline
\end{tabular}
ionic radii and oxygen parameter of the system $\mathrm{Li}_{0.5-\mathrm{x} / 2} \mathrm{Co}_{\mathrm{x}} \mathrm{Fe}_{2.5-\mathrm{x}} \mathrm{O}_{4}$

${ }^{10}$ Ostafiychuk B.K., Kaykan L.S., Kaykan J.S., Deputat B.Ya., \& Shevchuk O.V. Composition, Microstructure, and Electrical Properties Control of the Powders Synthesized by Sol-Gel Auto-Combustion Method Using Citric Acid as the Fuel. Nanoscale Research Letters. 2017. No. 12(1). URL: https://doi.org/10.1186/s11671017-1976-1. 
Figure 4 shows the change in the ionic radii of the system when replaced by cobalt ions.

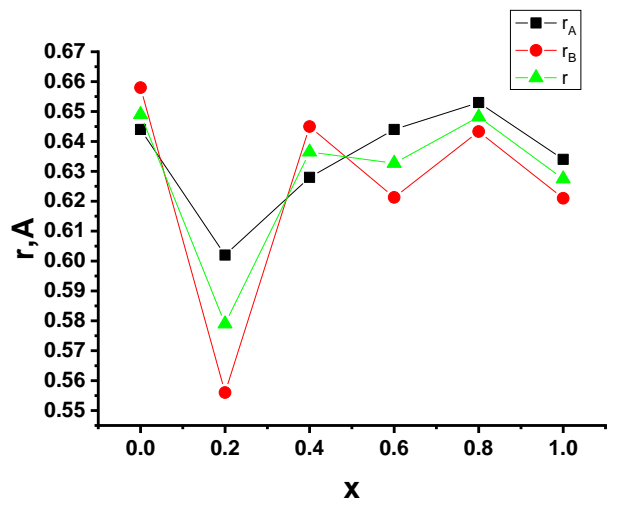

Fig. 4. Dependence of radii of cationic positions on the content of cobalt ions in the system $\mathrm{Li}_{0.5-\mathrm{x} / 2} \mathrm{Co}_{\mathrm{x}} \mathrm{Fe}_{2.5-\mathrm{x}} \mathrm{O}_{4}$

When replacing cobalt $x=0.2$, there is a decrease in the size of both $\mathrm{A}$ and $\mathrm{B}$ radii. With a further increase in the content of $\mathrm{x}$, the value of both radii gradually increases to $\mathrm{x}=0.8$, and then decreases again.

The theoretical value of the B-lattice constant was also calculated based on the Mazen equation ${ }^{11}$ :

$$
a_{t h}=\frac{8}{3 \sqrt{3}}\left[\left(r_{A}+R_{0}\right)+\sqrt{3\left(r_{B}+R_{0}\right)}\right],
$$

where $\mathrm{R}_{0}$ - radius of oxygen ions (1.32 $\AA$ ). The value of the oxygen parameter $\mathrm{u}$ was obtained from the results of the approximation of experimental X-ray diffraction patterns by the full-profile Rietveld refinement method. Slightly higher values of the experimental constant lattice than the theoretical one can obviously be explained by the small size of the crystallites. It is known ${ }^{12}$ that in the case of crystallite sizes $<100 \mathrm{~nm}$, the influence of the crystal surface is strongly manifested.

11 Sijo A.K., Jha V.K., Kaykan L.S., \& Dutta D.P. Structure and cation distribution in superparamagnetic NiCrFeO4 nanoparticles using Mössbauer study. Journal of Magnetism and Magnetic Materials. 2020. No. 497, 166047. URL: https://doi.org/10.1016/j.jmmm.2019.166047.

${ }^{12}$ Kaykan L.S., Kaykan J.S., Yaremiy I.P., Ugorchuk O.M., Deputat B.Y., \& Nykoliuk M.O. (2016). Synthesis, Structure and Dielectric Properties of Magnesiumsubstituted Lithium Ferrite. Journal of Nano- and Electronic Physics. 2016. No. 8(4(2)), 04066-1-04066-6. URL: https://doi.org/10.21272/jnep.8(4(2)).04066. 
The size of the coherent scattering (CSR) regions was determined from the half-width of the lines using the Scherrer formula

$$
D=\frac{k \lambda}{\beta \cos \theta},
$$

where $\lambda$ - wavelength of X-rays (1.54056A), $\theta$ - diffraction angle of the main reflexes, $\beta$ - peak width at half height (FWHM) i k-Scherrer's constant, which takes a value of 0.94 for particles close to spherical cubic symmetry ${ }^{13}$. However, the dependence $\cos \theta$ on $1 / \beta$ (Scherrer's dependence) does not approached by the line well (see insert to Fig. 5), indicating the presence of stresses in lattice. More precisely, these values are given by the Williamson Hall equation, which connects both the size of the crystallites and the stress in the lattice caused by the broadening of the peak:

$$
\beta=\beta_{\text {size }}+\beta_{\text {strain }}=\frac{k \lambda}{D \cos \theta}+4 \varepsilon \tan \theta,
$$

where $\varepsilon$ parameter of micro stress, and other symbols have the above meanings. Dependence $\beta \cos \theta$ on $4 \sin \theta(\mathrm{W}-\mathrm{H}-$ Williamson-Hall dependence, Fig. 5) is a set of points with a small scatter, indicating the homogeneity of the lattice stresses and, thus, the isotropic nature of the samples.

Moreover, the dependence shows a positive slope, which reflects the presence of elastic stresses in nanoparticles. The sizes of crystallites (grains) and values of microstresses of samples determined according to section and inclination of $\mathrm{W}-\mathrm{H}$ dependence are given in table 4.

As can be seen from the dependence (Fig. 6) with increasing cobalt ion content, the X-ray density increases. This phenomenon is obviously associated with an increase in the value of the lattice constant and the molar mass of the synthesized product. Moreover, the increase in the lattice constant has a weaker effect on the value of X-ray density and, as a result, there is an almost monotonic increase in the latter.

13 Paik S.W., Kim W.T., \& Lee S.H. A crystallographic and Mössbauer spectroscopic study of (Fe, Ni, Al)3-O4 spinel ferrite. Journal of Magnetism and Magnetic Materials, 1997. No. 169(1-2), pp. 145-150. URL: https://doi.org/10.1016/s0304-8853(96)00740-8. 

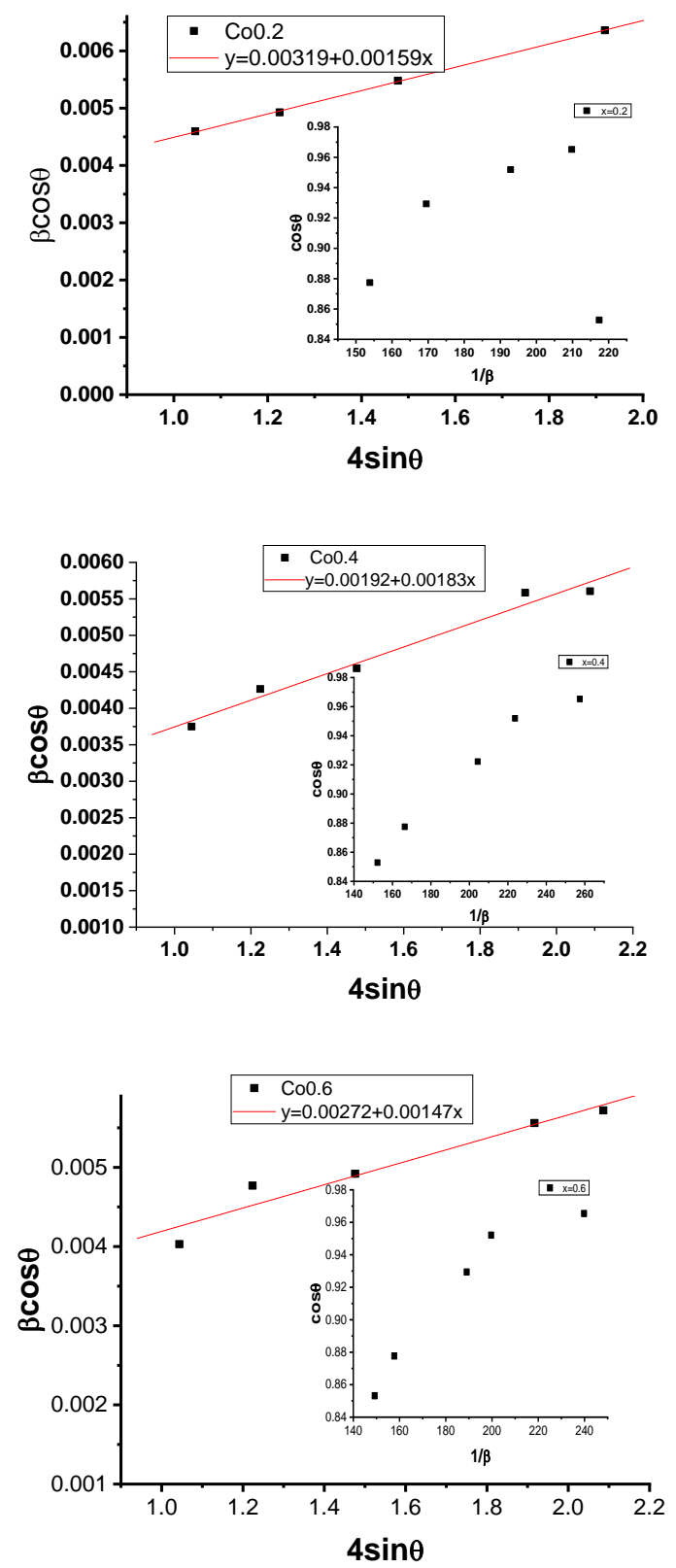

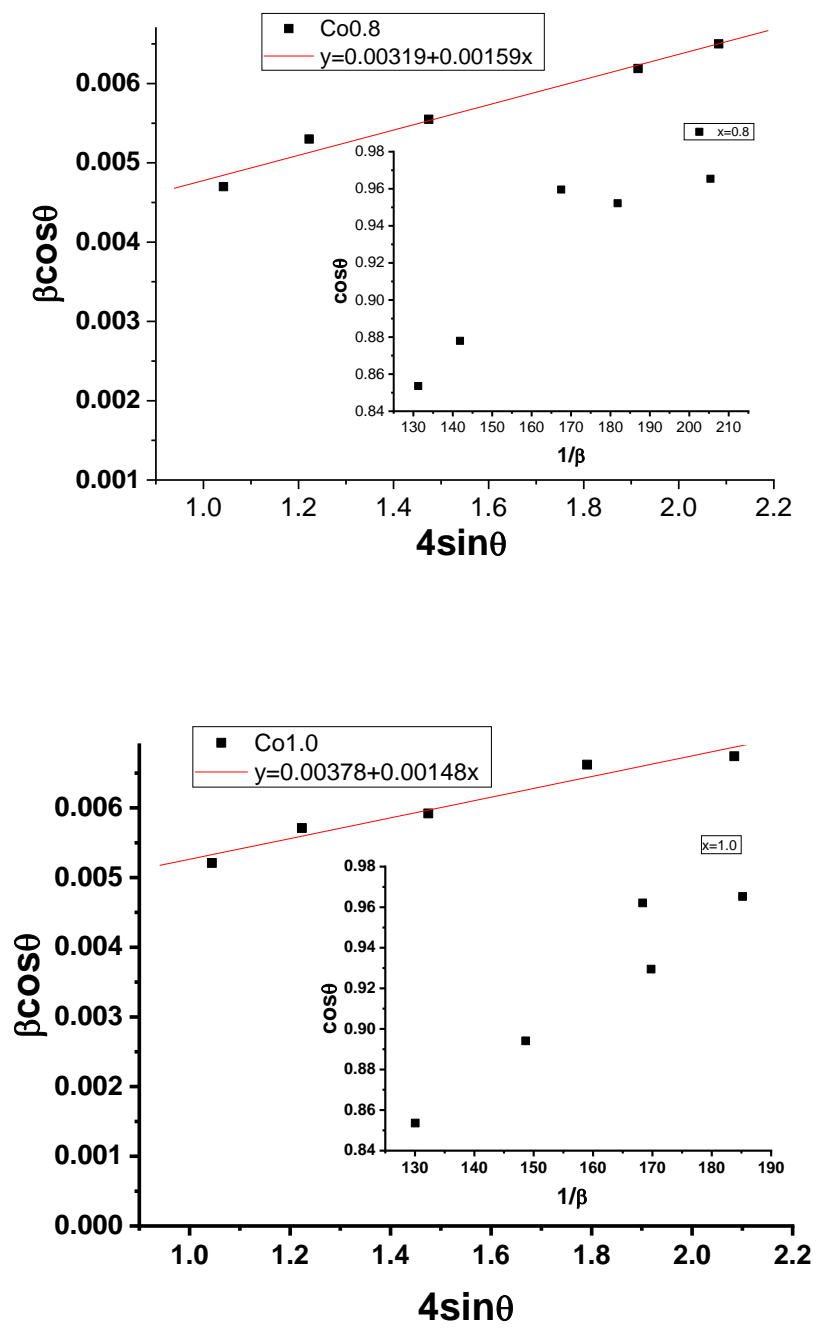

Fig. 5. Williamson-Hall (left) and Debye-Scherrer (right) dependences for $\mathrm{Li}_{0.5-\mathrm{x} / 2} \mathrm{Co}_{\mathrm{x}} \mathrm{Fe}_{2.5-\mathrm{x}} \mathrm{O}_{4}$ nanoparticles with different cobalt content 


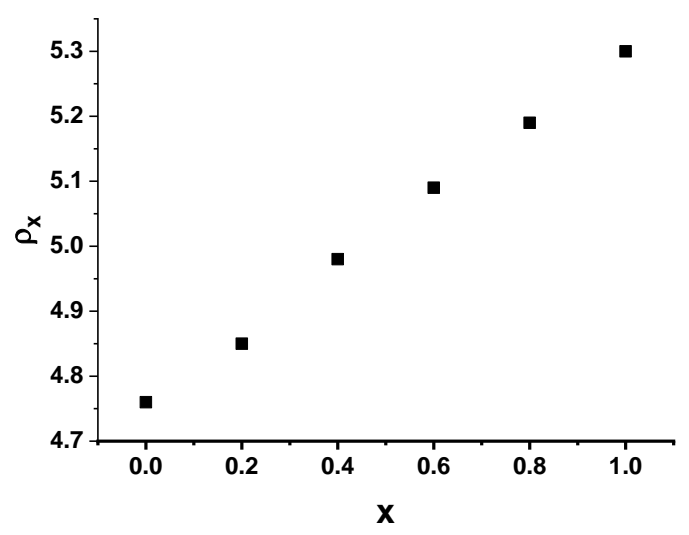

Fig. 6. Dependence of X-ray density on the content of cobalt ions

Table 4

\section{Structural and morphological characteristics of nanosized}

cobalt-substituted lithium ferrites $\mathrm{Li}_{0.5-\mathrm{x} / 2} \mathrm{Co}_{\mathrm{x}} \mathrm{Fe}_{2.5-\mathrm{x}} \mathrm{O}_{4}$

\begin{tabular}{|c|c|c|c|c|c|c|}
\hline $\begin{array}{c}\mathbf{C o}^{\mathbf{2 +}} \\
\mathbf{c o n t e n t ,}^{\mathbf{x}}\end{array}$ & $\begin{array}{c}\text { Molar } \\
\mathbf{m a s s}, \\
\mathbf{M}, \\
\mathbf{g} / \mathbf{m o l}\end{array}$ & $\begin{array}{c}\mathbf{X}-\mathbf{r a y} \\
\mathbf{d e n s i t y} \\
\mathbf{D}_{\mathbf{x}}, \\
\mathbf{g} / \mathbf{s m}^{\mathbf{3}}\end{array}$ & $\begin{array}{c}\text { Lattice } \\
\text { constant, } \\
\boldsymbol{a}, \mathbf{n m}\end{array}$ & $\begin{array}{c}\text { Crystallites } \\
\mathbf{s i z e}, \\
\mathbf{D}, \mathbf{n m}\end{array}$ & $\begin{array}{c}\text { Jump } \\
\text { length } \\
\mathbf{d}_{\text {A-A }}\end{array}$ & $\begin{array}{c}\text { Jump } \\
\text { length } \\
\mathbf{d}_{\mathbf{B}-\mathbf{B}}\end{array}$ \\
\hline 0.0 & 207.084 & 4.76 & 0.8330 & 42.3 & 0.1562 & 0.2945 \\
\hline 0.2 & 212.592 & 4.85 & 0.8351 & 45.5 & 0.1566 & 0.2953 \\
\hline 0.4 & 218.100 & 4.97 & 0.8351 & 36.4 & 0.1566 & 0.2953 \\
\hline 0.6 & 223.607 & 5.13 & 0.8356 & 55.2 & 0.1567 & 0.2954 \\
\hline 0.8 & 229.115 & 5.20 & 0.8365 & 36.4 & 0.1568 & 0.2957 \\
\hline 1.0 & 234.623 & 5.30 & 0.8377 & 36.4 & 0.1571 & 0.2962 \\
\hline errors & 0.001 & 0.01 & 0.0001 & 0.1 & 0.0001 & 0.0001 \\
\hline
\end{tabular}

As can be seen from the table, a clear trend of the influence of the content of the substituent element on the size of the crystallites is not observed. Slightly higher CSR values for samples $x=0.0(42 \mathrm{~nm})$ and $\mathrm{x}=0.6(55 \mathrm{~nm})$ are obviously related to the rate of the auto-combustion reaction, which depends on the metal/ fuel ratio ${ }^{14}$.

${ }^{14}$ Sileo E.E., Rotelo R., \& Jacobo S.E. Nickel zinc ferrites prepared by the citrate precursor method. Physica B: Condensed Matter, 2002. No. 320(1-4), pp. 257-260. URL: https://doi.org/10.1016/s0921-4526(02)00705-6. 


\section{Optimization of magnetic properties of nanosized lithium ferrites obtained by sol-gel auto-combustion method by substitution of cobalt ions}

Fig. 6 shows the Mössbauer spectra ${ }^{57} \mathrm{Fe}$ at temperature $293 \mathrm{~K}$ of $\mathrm{Li}_{0.5-\mathrm{x} / 2} \mathrm{Co}_{\mathrm{x}} \mathrm{Fe}_{2.5-\mathrm{x}} \mathrm{O}_{4}$, where $\mathrm{x}=0.0 ; 0.2 ; 0.4 ; 0.6 ; 0.8 ; 1.0$ obtained at a $\mathrm{pH}$ of the reaction medium equal to seven. All the obtained spectra are a superposition of three magnetically ordered components and two (for a system with $\mathrm{x}=0.4$ one) paramagnetic doublets.

The parameters of decoding of the Mössbauer spectra for different contents of cobalt ions are given in table 5 .

Table 5

Parameters of decoding of the Mössbauer spectra of the system $\mathrm{Li}_{\mathbf{0 . 5}-\mathrm{x} / \mathbf{2}} \mathrm{Co}_{\mathrm{x}} \mathrm{Fe}_{2.5-\mathrm{x} / \mathbf{2}} \mathrm{O}_{\mathbf{4}}$

\begin{tabular}{|c|c|c|c|c|c|c|}
\hline Sample & \multicolumn{6}{|c|}{ 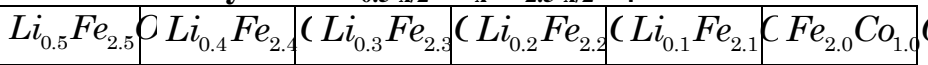 } \\
\hline Is & 0,2606 & 0,2471 & 0,3042 & 0,2588 & 0,2607 & 0,2592 \\
\hline Qs & $-0,0169$ & 0,0079 & $-0,0017$ & 0,0082 & 0,0147 & 0,0085 \\
\hline $\mathbf{H}_{\text {eff }}$ & 496,87 & 506.08 & 501,17 & 496,44 & 492,96 & 491,70 \\
\hline $\mathbf{S}$ & $\begin{array}{l}51,04 \\
\end{array}$ & 16,96 & 44,76 & 49,81 & 43,26 & 61,43 \\
\hline G & 0,4525 & 0,3112 & 0,2874 & 0,5071 & 0,4498 & 0,5690 \\
\hline Is & 0,3557 & 0,5551 & 0,4021 & 0,3888 & 0,3751 & 0,4020 \\
\hline Qs & 0,0080 & $-0,0106$ & $-0,0147$ & $-0,0209$ & $-0,0295$ & $-0,0117$ \\
\hline $\mathbf{H}_{\text {eff }}$ & 507,76 & 514,82 & 512,33 & 510,55 & 511,39 & 512,84 \\
\hline $\mathbf{S}$ & 40,29 & 14,31 & 38,29 & 30,19 & 31,30 & 23,34 \\
\hline G & 0,3500 & 0,2468 & 0,2928 & 0,4601 & 0,4860 & 0,4972 \\
\hline Is & 0.3356 & 0,1991 & 0,0775 & 0,4160 & 0,3422 & 0,6549 \\
\hline Qs & -0.504 & 0,0354 & $-0,0997$ & 0,0536 & $-0,0566$ & 0,3773 \\
\hline $\mathbf{H}_{\text {eff }}$ & 455.53 & 496,27 & 485,26 & 470,82 & 470,91 & 482,04 \\
\hline $\mathbf{S}$ & 8.67 & 63,16 & 14,68 & 18,23 & 20,32 & 9,46 \\
\hline G & 0.7760 & 0,6483 & 0,8838 & 103806 & 1,2216 & 0,4211 \\
\hline Is & & 0,0630 & 0,0489 & 0,3846 & 0,3589 & 0,3418 \\
\hline Qs & & 0,3569 & 0,3993 & 1,1134 & 0,7987 & 0,7457 \\
\hline $\mathbf{S}$ & & 3.53 & 2,27 & 0,32 & 4,93 & 4,21 \\
\hline G & & 0,3115 & 0,2406 & 0,1746 & 0,5090 & 0,4932 \\
\hline Is & & 0,0928 & & 0,3662 & 0,4446 & 0,3489 \\
\hline Qs & & 1,0964 & & 0,5305 & 2,1123 & 1,8486 \\
\hline $\mathbf{S}$ & & 1.16 & & 1,44 & 0,19 & 1,55 \\
\hline G & & 0,2450 & & 0,3828 & 0,1746 & 0,4360 \\
\hline
\end{tabular}



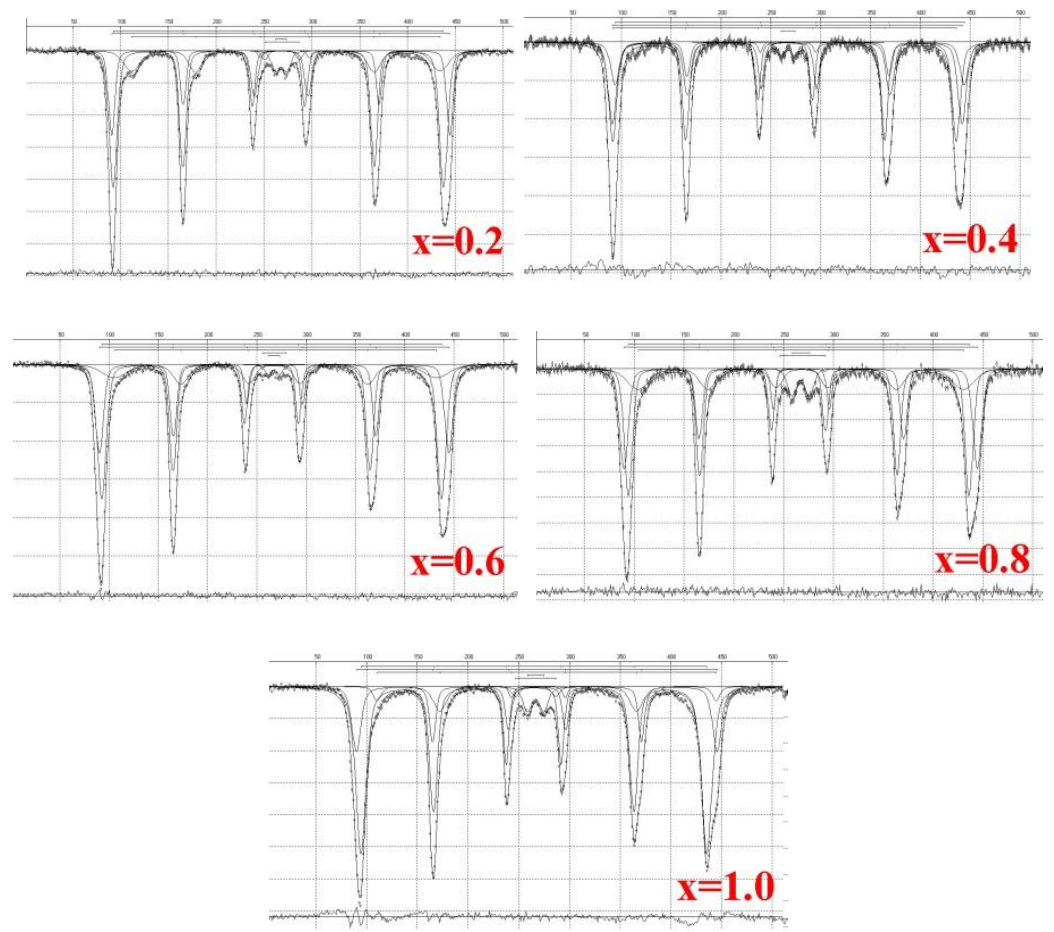

Figure 7. Mössbauer spectra ${ }^{57} \mathrm{Fe}$ at temperature 293K of $\mathrm{Li}_{0.5-\mathrm{x} / 2} \mathrm{Co}_{\mathrm{x}} \mathrm{Fe}_{2.5-\mathrm{x} / 2} \mathrm{O}_{4}$ systems, where $\mathrm{x}=0.2 ; 0.4 ; 0.6 ; 0.8 ; 1.0$, obtained at a $\mathrm{pH}$ of the reaction medium equal to seven

Magnetic fields with relatively large values are responsible for the $\mathrm{A}$ and $\mathrm{B}$ environment of ${ }^{57} \mathrm{Fe}$ nuclei. These values change with increasing concentration of the substituent element. In Fig. 7 shows the dependences of the magnitude of the ${ }^{57} \mathrm{Fe}$ magnetic fields in the $\mathrm{A}, \mathrm{H}_{\mathrm{A}}$, and $\mathrm{B}, \mathrm{H}_{\mathrm{B}}$, environment. The increase in the value of the effective magnetic fields in the case of $x=0.2$ can obviously be explained by the peculiarities of the structure. As can be seen from Fig. 4 for this composition $(\mathrm{x}=0.2)$ there is a sharp decrease in the radii of both cavities, which leads to a decrease in the distances of the interlattice superchange interaction and, as a consequence, an increase in the values of the effective magnetic fields for this composition. 


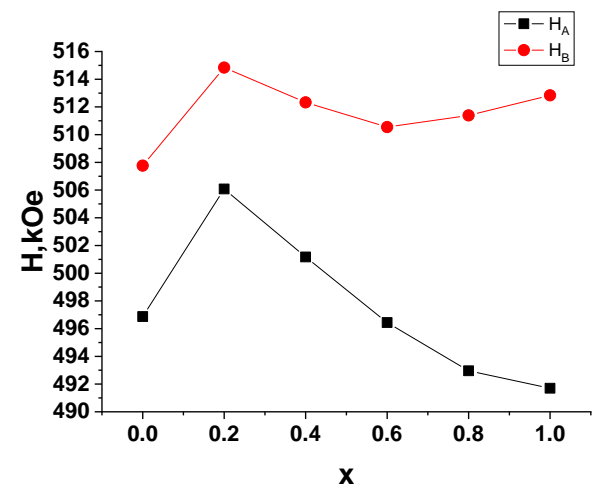

Fig. 8. Dependence of magnetic fields $\mathrm{H}_{\mathrm{A}}$ and $\mathrm{H}_{\mathrm{B}}$ on cobalt content in the system $\mathrm{Li}_{0.5-\mathrm{x} / 2} \mathrm{Co}_{\mathrm{x}} \mathrm{Fe}_{2.5-\mathrm{x} / 2} \mathrm{O}_{4}$

The presence of the third magnetically ordered component on the Mössbauer spectra with values of effective magnetic fields several tens of $\mathrm{kE}$ smaller, we attributed to the ${ }^{57} \mathrm{Fe}$ nuclei, located in the surface layer. The decrease in the effective magnetic fields on the ${ }^{57} \mathrm{Fe}$ nuclei is due to the different number of indirect exchange bonds for internal and surface iron ions, which are deprived of a significant part of such bonds in $^{15}$. The idea of our proposed method for estimating the size of crystallites is based on the allocation of the spectrum, the characteristics of which are given above. In other words, a subspectrum whose magnitude above the exchange field is several tens of kilooersted is smaller than the magnitude above the exchange fields due to iron ions in A and B lattices. This area, taking into account the crystal structure of the spinel, can include the surface layer of iron, the environment of which differs from the environment of the internal iron ions. Reduction of effective magnetic fields on ${ }^{57} \mathrm{Fe}$ nuclei for surface ions is approximately $50 \mathrm{kOe}$. This decrease is due to the different number of indirect exchange bonds for internal and external iron ions, which are

15 Yadav R.S., Havlica J., Hnatko M., Šajgalík P., Alexander C., Palou M., Bartoníčková E., Boháč M., Frajkorová F., Masilko J., Zmrzlý M., Kalina L., Hajdúchová M., \& Enev V. Magnetic properties of Co 1-x Zn x Fe 2 O 4 spinel ferrite nanoparticles synthesized by starch-assisted sol-gel auto-combustion method and its ball milling. Journal of Magnetism and Magnetic Materials. 2015. No. 378, pp. 190-199. URL: https://doi.org/10.1016/j.jmmm.2014.11.027. 
deprived of a significant part of such bonds. In ${ }^{16,17}$, based on the method of molecular orbitals in the approximation of the linear combination of atomic orbitals, estimates of the contributions to the effective magnetic field from each of the indirect exchange bonds for the $\mathrm{Fe}^{3+}$ ion in the spinel structure were made. These contributions for positions A and B were 8 and 12kOe, respectively. Since for iron ions in the A position in the immediate cationic environment there are 12 iron ions in the $\mathrm{B}$ position, and for iron ions in the $\mathrm{B}$ position - six iron ions in the $\mathrm{A}$ position, the absence of half of the bonds for surface atoms should reduce the effective magnetic fields, several tens of $\mathrm{kOe}$, which is consistent with experimental data. If we proceed from the approximation of the spherical shape of the particles, then the results of decoding of the Mössbauer spectra it is possible to identify that part $S_{e}$ of the total area of the Mössbauer spectrum $\mathrm{S}=\mathrm{S}_{\mathrm{i}}+\mathrm{S}_{\mathrm{e}}$, which corresponds to the surface atoms of iron, then from these data we can estimate the particle size. Indeed, taking the volumes of the surface and inner regions are equal, respectively $V_{e}=4 \pi r^{2} \Delta r$ and $V_{i}=4 / 3 \pi r^{3}$, where $r$ particle radius, $\Delta r-$ the thickness of the surface area will be approximately

$$
\frac{S_{e}}{S_{i}}=\frac{3 \Delta r}{r} .
$$

Taking into account the values of the areas under the subspectra, it is possible to estimate the particle size. This took into account the assumption of the spherical shape of the particles. Table 4, along with data on the size of the crystallites obtained from X-ray data, shows the calculated data from Mössbauer experiments. The close values of both results indicate the validity of this method of assessment.

A paramagnetic doublet with a small value of quadrupole splitting also serves as a criterion for estimating the size of crystallites. Fig. 9 shows the change in the size of the crystallites and the area of the paramagnetic doublet from the content of cobalt ions.

16 Lakshman A., Subba Rao P.S.V., \& Rao K.H. (2006). Mössbauer spectroscopic analyses of $\mathrm{Mg} 0.9 \mathrm{Cu} 0.1 \mathrm{Mn} 0.05 \mathrm{CrxFe} 1.95-\mathrm{xO} 4$ spinel ferrites. Materials Letters. 2006. No. 60(1), pp.7-10. URL: https://doi.org/ 10.1016/j.matlet.2005.07.043.

${ }^{17}$ Gabal M.A., Al-Thabaiti S.A., El-Mossalamy E.H., \& Mokhtar M. Structural, magnetic and electrical properties of Ga-substituted NiCuZn nanocrystalline ferrite. Ceramics International. 2010. No. 36(4), pp. 1339-1346. URL: https://doi.org/ 10.1016/j.ceramint.2010.01.021. 
As can be seen from the figure, there is an inverse relationship between these parameters, which can also serve as a criterion for estimating the size of the synthesized ferrite. As the size of the crystallites decreases, the area under the doublet increases.

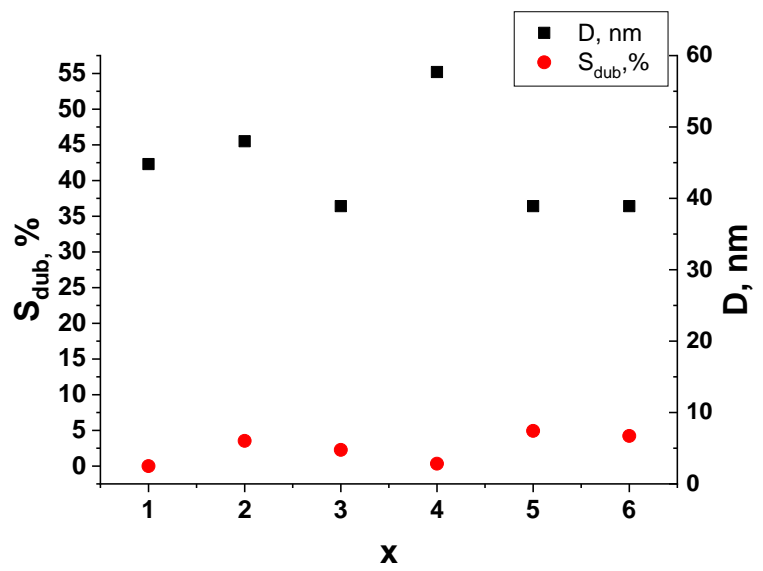

Fig. 9. Dependence of crystallite size and area under a paramagnetic doublet with a small value of quadrupole splitting on the content of cobalt ions

Thus, the method of obtaining and the chemical composition of the synthesized product makes a significant contribution to changing the properties of the material, which indicates the possibility of systematic modification of the properties of the material given the peculiarities of its application.

\section{CONCLUSIONS}

Cobalt-substituted lithium ferrites of general composition $\mathrm{Li}_{0.5-\mathrm{x} / 2} \mathrm{Co}_{\mathrm{x}} \mathrm{Fe}_{2.5-\mathrm{x}} \mathrm{O}_{4}$, where $\mathrm{x}=0.0 ; 0.2 ; 0.4 ; 0.6 ; 0.8 ; 1.0$, were synthesized by the method of sol-gel auto-combustion. It is shown that the structure of the synthesized material is sensitive to the cobalt content. There is a transformation of the structure from ordered spinel $\mathrm{P}_{3} 32$ to disordered $\mathrm{Fd} 3 \mathrm{~m}$, which is due to the localization of some cobalt ions in the B-site. The sizes of crystallites of the received systems are in the range of $36-43 \mathrm{~nm}$. 
As the concentration of cobalt in lithium ferrites synthesized by the sol-gel method of auto-combustion increases, the saturation magnetization increases to $\mathrm{x} \leq 0.8$ and then decreases. This is because $\mathrm{Co}^{2+}$ ions with high magnetic moment replace non-magnetic $\mathrm{Li}^{+}$ions in synthesized Li-Co ferrite samples. However, in the case of pure cobalt ferrites $(\mathrm{x}=1.0), \mathrm{Co}^{2+}$ ions replace only $\mathrm{Fe}^{2+}$ ions resulting in a decrease in magnetization in pure cobalt ferrite. With increasing substitution of cobalt in Li-Co ferrite, the material turns from magnetically soft to magnetically hard.

As a result of synthesis by the sol-gel method of auto-combustion at different $\mathrm{pH}$ values of the reaction medium, single-phase spinel ferrites of the $\mathrm{Fd} 3 \mathrm{~m}$ space group were obtained. It was found that the reaction medium significantly affects the morphology of the obtained product. Thus, the size of the crystallites obtained at $\mathrm{pH}=7$ is in the range of $\sim 20 \mathrm{~nm}$, while at $\mathrm{pH}=3$ and $\mathrm{pH}=9$ the crystallites are relatively larger and equal to $\sim 25-35 \mathrm{~nm}$. This is due to the effect of ammonia, which increases the chelation of metal cations with citrates and promotes the formation of a porous three-dimensional (3D) structure in nitrate-citrate xerogels. Increasing the temperature and rate of exothermic reaction at $\mathrm{pH}=7$ prevents agglomeration of crystallites.

The Mössbauer ${ }^{57} \mathrm{Fe}$ spectra of the $\mathrm{Li}_{0.2} \mathrm{Fe}_{2.2} \mathrm{Co}_{0.6} \mathrm{O}_{4}$ system obtained at $\mathrm{pH}$ values equal 3, 7, 9 also show a dependence on the $\mathrm{pH}$ of the reaction medium, which is caused by both morphology and crystallite size, because changes in structural parameters cause corresponding changes in the magnetic microstructure. An increase in the lattice parameter at $\mathrm{pH}=7$ causes an increase in the lengths of the Me-Me and $\mathrm{Me}-\mathrm{O}$ bonds that are responsible for the magnetic interaction in spinel ferrites. Thus, the synthesis conditions, and in particular the $\mathrm{pH}$ value of the reaction medium, play an important role in the formation of the structure and properties of the material synthesized by the sol-gel method of auto-combustion.

\section{SUMMARY}

Polycrystalline nanosized ferrites have good chemical stability and high magnetic characteristics, which depend on a number of factors, such as method of preparation, type and amount of replacement element, heat treatment, etc. Lithium and substituted lithium ferrites with a spinel structure have proven to be a good substitution of garnets in microwave devices due to their low cost, high resistance, low eddy current losses 
and at the same time high magnetic characteristics, such as saturation magnetization, residual magnetization, high Curie temperature.

In this work nanoparticles of lithium spinel ferrites $\left(\mathrm{Li}_{0.5-\mathrm{x} / 2} \mathrm{Co}_{\mathrm{x}} \mathrm{Fe}_{2.5-}\right.$ $\left.{ }_{\mathrm{x}} \mathrm{O}_{4}, \mathrm{x}=0.0 ; 0.2 ; 0.4 ; 0.6 ; 0.8 ; 1.0\right)$ were successfully synthesized by a modified method of sol-gel auto-combustion. Lithium nitrate, cobalt nitrate and iron nitrate were selected as the source of monovalent and divalent $\left(\mathrm{Li}^{+} \mathrm{i} \mathrm{Co}^{2+}\right)$ and trivalent $\left(\mathrm{Fe}^{3+}\right)$ cations, respectively, and as a reducing agent (fuel) selected citric acid. Was studied the influence of cobalt ions on the morphology, structural, electrical and magnetic properties of $\mathrm{Li}_{0.5-\mathrm{x} / 2} \mathrm{Co}_{\mathrm{x}} \mathrm{Fe}_{2.5-\mathrm{x}} \mathrm{O}_{4}$ spinel.

The observed results showed that the final product is a cubic spinel with a spherical morphology of nanoparticles. Moreover, with increasing cobalt concentration in lithium ferrites synthesized by the sol-gel method of auto-combustion, the saturation magnetization increases to $\mathrm{x} \leq 0.8$, and then decreases. This is because $\mathrm{Co}^{2+}$ ions with high magnetic moment replace non-magnetic $\mathrm{Li}^{+}$ions in synthesized $\mathrm{Li}-\mathrm{Co}$ ferrite samples.

\section{REFERENCES}

1. De Fazio E., Bercoff P.G., \& Jacobo S.E. Electromagnetic properties of manganese-zinc ferrite with lithium substitution. Journal of Magnetism and Magnetic Materials, 2011. No. 323(22), pp. 2813-2817. URL: https://doi.org/10.1016/j.jmmm.2011.06.022.

2. Liu J., Mei Y., Liu W., Li X., Hou F., \& Lu G.-Q. Effects of sintering temperature on properties of toroid cores using $\mathrm{NiZnCu}$ ferrites for power applications at $>1 \mathrm{MHz}$. Journal of Magnetism and Magnetic Materials. 2018. No. 454, pp. 6-12. URL: https://doi.org/10.1016/j.jmmm.2018.01.031.

3. Singh Yadav R., Kuřitka I., Havlica J., Hnatko M., Alexander C., Masilko J., Kalina L., Hajdúchová M., Rusnak J., \& Enev, V. Structural, magnetic, elastic, dielectric and electrical properties of hot-press sintered Co1-xZnxFe2O4 $(\mathrm{x}=0.0,0.5)$ spinel ferrite nanoparticles. Journal of Magnetism and Magnetic Materials, 2018. No. 447, pp. 48-57. URL: https://doi.org/10.1016/j.jmmm.2017.09.033.

4. Han D., Zhang J., Liu P., Li G., An L., \& Wang S. Preparation of high-quality transparent Al-rich spinel ceramics by reactive sintering. Ceramics International. 2018. No. 44(3), pp. 3189-3194. URL: https://doi.org/10.1016/j.ceramint.2017.11.089.

5. Rahaman Md.D., Nusrat T., Maleque R., \& Hossain A.K.M.A. Investigation of structural, morphological and electromagnetic properties 
of $\mathrm{Mg} 0.25 \mathrm{Mn} 0.25 \mathrm{Zn} 0.5-\mathrm{xSrxFe} 2 \mathrm{O} 4$ ferrites. Journal of Magnetism and Magnetic Materials. 2018. No. 451, pp. 391-406. URL: https://doi.org/10.1016/j.jmmm.2017.11.066.

6. Sun L., Guo J., Zhang R., Cao E., Zhang Y., Hao W., \& Ju L. Influence of $\mathrm{Cu} 2+$ doping on the structure, dielectric and magnetic properties of $\mathrm{NiFe} 2 \mathrm{O} 4$ prepared by the sol-gel method. Journal of Magnetism and Magnetic Materials. 2018. No. 449, pp. 545-551. URL: https://doi.org/10.1016/j.jmmm.2017.10.104.

7. Elshahawy A.M., Mahmoud M.H., Makhlouf S.A., \& Hamdeh H.H. Role of $\mathrm{Cu} 2+$ substitution on the structural and magnetic properties of $\mathrm{Ni}$-ferrite nanoparticles synthesized by the microwavecombustion method. Ceramics International. 2015. No. 41(9), pp. 11264-11271. URL: https://doi.org/10.1016/j.ceramint.2015.05.079.

8. Ahmed M.A., Mansour S.F., \& Afifi M. Structural and electrical properties of nanometric $\mathrm{Ni}-\mathrm{Cu}$ ferrites synthesized by citrate precursor method. Journal of Magnetism and Magnetic Materials. 2012. № 324(1), pp. 4-10. URL: https://doi.org/10.1016/j.jmmm.2011.07.010.

9. Sijo A.K. Influence of fuel-nitrate ratio on the structural and magnetic properties of $\mathrm{Fe}$ and $\mathrm{Cr}$ based spinels prepared by solution selfcombustion method. Journal of Magnetism and Magnetic Materials. 2017. No. 441, pp. 672-677. URL: https://doi.org/10.1016/ j.jmmm.2017.06.060; M.A. Dar, Dinesh Varshney. Effect of d-block element $\mathrm{Co} 2+$ substitution on structural, Mössbauer and dielectric properties of spinel copper ferrites. Journal of Magnetism and Magnetic Materials. 2017. V. 436, pp.101-112.

10.Ostafiychuk B.K., Kaykan L.S., Kaykan J.S., Deputat B.Ya., \& Shevchuk O.V. Composition, Microstructure, and Electrical Properties Control of the Powders Synthesized by Sol-Gel Auto-Combustion Method Using Citric Acid as the Fuel. Nanoscale Research Letters, 2017. No. 12(1). URL: https://doi.org/10.1186/s11671-017-1976-1.

11.Sijo A.K., Jha V.K., Kaykan L.S., \& Dutta D.P Structure and cation distribution in superparamagnetic $\mathrm{NiCrFeO} 4$ nanoparticles using Mössbauer study. Journal of Magnetism and Magnetic Materials. 2020. No. 497, 166047. URL: https://doi.org/10.1016/j.jmmm.2019.166047.

12. Kaykan L.S., Kaykan J.S., Yaremiy I.P., Ugorchuk O.M., Deputat B.Y., \& Nykoliuk M.O. Synthesis, Structure and Dielectric Properties of Magnesium-substituted Lithium Ferrite. Journal of Nanoand Electronic Physics. 2016. No. 8(4(2)), 04066-1-04066-6. URL: https://doi.org/ 10.21272/jnep.8(4(2)).04066. 
13.Paik S.W., Kim W.T., \& Lee S.H. A crystallographic and Mössbauer spectroscopic study of (Fe, Ni, Al)3-O4 spinel ferrite. Journal of Magnetism and Magnetic Materials, 1997. No. 169(1-2), pp. 145-150. URL: https://doi.org/10.1016/s0304-8853(96)00740-8.

14. Sileo E.E., Rotelo R., \& Jacobo S.E. (2002). Nickel zinc ferrites prepared by the citrate precursor method. Physica B: Condensed Matter, 2002. No. 320(1-4), pp. 257-260. URL: https://doi.org/10.1016/s09214526(02)00705-6.

15. Yadav R.S., Havlica J., Hnatko M., Šajgalík P., Alexander C., Palou M., Bartoníčková E., Boháč M., Frajkorová F., Masilko J., Zmrzlý M., Kalina L., Hajdúchová M., \& Enev V. Magnetic properties of Co 1-x $\mathrm{Zn}$ x Fe $2 \mathrm{O} 4$ spinel ferrite nanoparticles synthesized by starch-assisted sol-gel auto-combustion method and its ball milling. Journal of Magnetism and Magnetic Materials, 2015. No. 378, pp. 190-199. URL: https://doi.org/10.1016/j.jmmm.2014.11.027.

16.Lakshman A., Subba Rao P.S.V., \& Rao K.H. Mössbauer spectroscopic analyses of $\mathrm{Mg} 0.9 \mathrm{Cu} 0.1 \mathrm{Mn} 0.05 \mathrm{CrxFe} 1.95-\mathrm{xO} 4$ spinel ferrites. Materials Letters, 2006. No. 60(1), pp. 7-10. URL: https://doi.org/10.1016/j.matlet.2005.07.043.

17. Gabal M.A., Al-Thabaiti S.A., El-Mossalamy E.H., \& Mokhtar M. Structural, magnetic and electrical properties of Ga-substituted $\mathrm{NiCuZn}$ nanocrystalline ferrite. Ceramics International, 2010. No. 36(4), pp. 1339-1346. URL: https://doi.org/10.1016/j.ceramint.2010.01.021.

\section{Information about authors: Mazurenko Yu. S.,}

Candidate of Physical and Mathematical Sciences, Assistant Professor at the Department of Medical Informatics,

Medical and Biological Physics

Ivano-Frankivsk National Medical University 2, Halytska str., Ivano-Frankivsk, 76018, Ukraine

Kaykan L. S.,

Candidate of Physical and Mathematical Sciences, Researcher at the Joint Educational and Scientific Laboratory of Physics Magnetic Tapes

G. V. Kurdyumov Institute for Metal Physics of the National Academy of Sciences of Ukraine 36, Academician Vernadsky blvd, Kyiv, 03142, Ukraine 